
Verlag von VEIT \& COMP. in Leipzig.

\section{ANLEITUNG ZUR DARSTELLUNG \\ CHEMISCHER ANORGANISCHER PRÄPARATE}

für Chemiker und Pharmazeuten.

Dr. Reinhart Blochmann,

Professor der Chemie an der Universităt Königsberg i. Pr.

Mit zahlreichen Abbildungen.

8. 1895. geb. in Ganzleinen $2 \mathscr{N} 20 \%$.

\section{U'BER DIE LOSSUNGEN.}

Einführung in die Theorie der Lösungen, die Dissoziationstheorie und das Massenwirkungsgesetz.

Nach Vorträgen, gehalten im Physiologischen Vereine und im Vereine

zur Beförderung des naturwissenschaftlichen Unterrichtes zu Breslau.

Dr. W. Herz,

Privatdozenten for Chemie an der Universitat Breslau.

gr. 8. 1903. geh. 1 th 40 Fु.

\section{PRAKTISCHER LEITFADEN

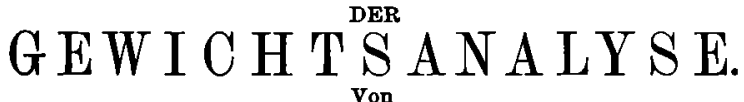

Dr. Paul Jannasch,

Professor an der Universität Heidelberg.

Zweite, verbesserte und vermehrte Auflage.

Mit zahlreichen Abbildungen im Text.

gr. 8. 1904. geb. in Ganzleinen 8 \%.

Vor anderen analytischen Büchern zeichnet sich das vorliegende durch die auBerordentlich genaue Beschreibung der Arbeitsmethoden aus.

Die zweite Auflage ist durch Aufnahme neuer Methoden sehr erweitert worden, besonders auch durch vom Verfasser selbst ausgearbeitete Methoden. Das Buch leitet in ansgezeichneter Weise zum selbständigen Arbeiten an und ist daher besonders geeignet, als Übungsbuch in analytischen Laboratorien eingeführt zu werden. Auch dem im praktischen Leben stehenden Chemikerist es ein guter Berater bei analytischen Arbeiten.

\section{GRUNDRISS \\ DER \\ PHYSIKALISCHEN KRYSTALLOGRAPHIE. Von \\ Dr. Theodor Liebisch,}

o. ö. Professor der Mineralogie an der Universität Göttingen.

Mit 898 Figuren im Text.

Lex. 8. 1896. geh. $13 \mathscr{H} 40 \mathscr{7}$, geb. in Halbfr. $15 \mathscr{M} 40$.

ELEKTROCHEMIE.

Ihre Geschichte und Lehre. von

Wilhelm Ostwald.

Mit 260 Nachbildungen geschichtlicher Originalfiguren.

Lex. 8. 1896. geh. $28 \mathscr{K}$, eleg. geb. $30 \mathscr{K}$. 
Verlag von VEIT \& COMP. in Leipzig.

\section{QUALITATIVE ANALYSE UNORGANISCHER SUBSTANZEN. Von}

Dr. Heinrich Biltz,

Professor der Chemie an der Universitat Kiel.

Mit sieben Figuren.

gr. 8. 1900. geb. in Ganzleinen 1 A6 $80 \mathscr{F}$.

\section{ANLEITUNG ZUR GESTEINSANALYSE. Von}

Dr. Max Dittrich,

a. o. Professor an der Universitar Heldelberg.

Mit funf Figuren.

gr. 8. 1905. geb. in Ganzleinen 3 $1600 \%$.

\section{PRAKTIKUM \\ DES \\ ANORGANISCHEN CHEMIKERS.}

Einführung in die anorganische Chemie auf experimenteller Grundlage.

$$
\text { Von }
$$

Dr. Emil Knoevenagel,

Professor an der Universitat Heidelberg.

Mit zahlreichen Figuren, Tabellen und sieben Tafeln.

gr. 8. 1901. geb. in Ganzleinen $7.4680 \%$.

Das „Praktikum" ist dazu bestimmt, als Einführung in die anorganische Clemie zu dienen. Es unterscheidet sich von anderen Werken Whnlicher Tendenz wesentlich dadurch, daB es nicht lediglich eine analytische Chemie darstellt, sondern neben der Beschreibung der Arbeitsmethoden und einzelner. Versuche auch die dazu gehörigen theoretischen Erläuterungen enthält. Das "Praktikum" beginnt mit der Entwickelung der einfachsten chemischen Begriffe, soweit dieselben für die Analyse von Bedeutung sind, behandelt bei den einzelnen Elementen kurz deren Eigenschaften und bespricht ausführlich die analytisch wichtigen Verbindungen.

\section{LOGARITHMISCHE RECHENTAFELN FÜR CHEMIKER.}

Im Einverständnis mit der Atomgewichtskommission der Deutschen chemischen Gesellschaft

für den Gebrauch im Unterrichtslaboratorium

und in der Praxis berechnet und mit Erläuterungen versehen

Dr. F. ${ }_{\text {W. }}^{\text {von }}$ Küster,

Professor der Chemle an der Bergakademie Clausthal, Vorstand des Kgl. Betriebslaboratoriums daselbst.

Vierte, neu berechnete und erweiterte Auflage.

12. 1904. geb. in Ganzleinen $2 \%$. 
Verlag von VEIT \& COMP. in Leipzig.

\section{DIE DRAHTLOSE TELEGRAPHIE}

auf Grund eigner praktischer Erfahrungen.

Von

Dr. phil. Gustav Eichhorn.

Mit zahlreichen Figuren.

gr. 8. 1904. geh. $5 \mathscr{H}$, geb. in Ganzleinen $6 \mathscr{H}$.

LEHRBUCH DER ORGANISCHEN CHEMIE

für Studierende

an Universitäten und Technischen Hochschulen.

Von

Dr. A. F. Holleman,

o. Professor der Chemio an der Universitât Groningen.

Dritte, verbesserte Auflage.

Mit zahlreichen Abbildungen.

gr. 8. 1904. gebunden in Ganzleinen 10 A6.

\section{DIE \\ PRAXIS DES ORGANISCHEN CHEMIKERS.}

Von

Dr. Ludwig Gattermann,

o. Professor der Chemie an der Univorsitat Freiburg i. B.

Sechste, verbesserte und vermehrte Auflage.

Mit 91 Abbildungen im Text.

gr. 8. 1904. geb. in Ganzleinen 7

\section{GESCHICHTE DER CHEMIE}

von den ältesten Zeiten bis zur Gegenwart.

Zugleich Einfuhhrung in das Studium der Chemie.

Von

Dr. Ernst von Meyer,

o. Professor der Chemie an der Technischen Hochschule zu Dresden.

Dritte, verbesserte und vermehrte Auflage.

gr. 8. 1905. geh. $11 \%$, geb. in Ganzleinen $12 \mu$.

„Eine stets wiederkehrende Erfahrung als Forscher wie als Lehrer hat mich iiberzeugt, daß es kein wirksameres Mittel xur Belebung und

Vertiefung des Studiums gibt, als das Eindringen in das geschichtliche Werden der Probleme." Wilhelm Ostwald.

Zahlreiche Studierende der Chemie hat diese Geschichte der Entwickelung der chemischen Wissenschaft chemisch denken gelehrt. Aber auch der erfahrene Praktiker wird aus einem Buche Nutzen ziehen, das in anziehender Form den weiten Weg schildert, den die Chemie zurücklegen muBte, ehe sie zu ihrem heutigen Besitzstand gelangte. 



\section{LEHRBUCH}

\section{DER \\ C H E M I E}

voN

DR. A. F. HOLLEMAN,

O. PROFESSOR DER CHEMIE AN DER UNIVERSITÄT GRONINGEN.

AUTORISIERTE DEUTSCHE AUSGABE.

UNORGANISCHER TEIL.

DRITTE, VERBESSERTE AUFLAGE.

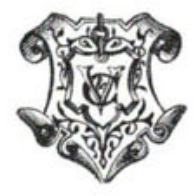

LEIPZIG

VERLAG VON VEIT \& COMP. 


\title{
LEHRBUCH
}

\author{
DER

\section{UNORGANISCHEN CHEMIE} \\ FÜR STUDIERENDE \\ AN UNIVERSITÄTEN UND TECHNISCHEN HOCHSCHULEN \\ vON \\ DR. A. F. HOLLEMAN, \\ O. PROFEgSOR DER CHEMIE AN DER UNIVkRgITÄT GRONINAEN.
}

DRITTE, VERBESSERTE AUFLAGE.

MIT ZAHLREICHEN ABBIDUNGEN UND ZWEI TAFELN.

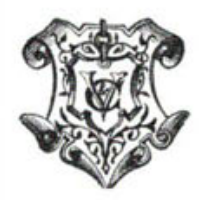

LEIPZIG

VERLAG VON VEIT \& COMP. 
Die Elnfubrung dieser deutschen Originalausgabe nach Holland ist untersagt. 